\title{
Actividades económicas primarias predominantes en la Reserva Paisajística Nor Yauyos Cochas, Perú. Caso: distritos de Huancaya, Alis y Vitis
}

\author{
Predominant economic primary activities in the Nor Yauyos Cochas Landscape \\ Reserve, Perú. Case: Huancaya, Alis and Vitis districts
}

Wilfredo Bulege Gutiérrez

Universidad Continental

\section{RESUMEN}

Objetivos: Determinar las actividades económicas primarias predominantes en los distritos de Huancaya, Alis y Vitis de la provincia de Yauyos, región Lima, ubicados en la zona de aprovechamiento directo de la Reserva Paisajística Nor Yauyos Cochas. Métodos: Es una investigación de tipo básica, alcance descriptiva, diseño transversal, en el proceso de recolección de datos se aplicó un cuestionario estructurado a una muestra no probabilística de 79 personas con edades mayores a 18 años entre varones y mujeres de los distritos de Huancaya, Alis y Vitis, quienes participaron en un evento académico sobre "Econegocios para el desarrollo" organizado por Grupo de Proyección Social "Eco-negocios" de la Universidad Continental, este mismo instrumento se aplicó a personas ubicadas circunstancialmente en la vía pública de los mencionados distritos hasta completar el tamaño muestral de 79. Resultados: El $42 \%$ manifiesta que se dedica principalmente a la agricultura; $37 \%$, a la ganadería; $5 \%$, a la acuicultura; $5 \%$, a la artesanía y un $11 \%$ a otras actividades entre las cuales se puede identificar el comercio, servicios turísticos, minería, etc. Las personas cuya actividad principal es la agricultura manifestaron que el $44 \%$ de su producción es maíz; $28 \%$, papa; $23 \%$, trigo y $5 \%$ otros productos. Las personas dedicadas a la ganadería manifestaron que el $41 \%$, cría camélidos; $28 \%$, vacunos; $14 \%$, ovinos y un $17 \%$, otros. Conclusiones: La población de los distritos de Huancaya, Alis y Vitis dedican como una de sus actividades económicas de mayor

predominancia a la agricultura, luego la ganadería, siendo este un indicador importante que caracteriza a estos distritos como zonas con una economía basada en actividades primarias, es decir extractiva de recursos naturales y aprovechada prioritariamente para autoconsumo y su comercialización.

Palabras clave: actividades económicas, predominantes, reserva paisajística, Nor Yauyos Cochas, Huancaya, Alis, Vitis.

1 Lic. en Pedagogía y Humanidades, Mg. en Administración, Candidato a Doctor en Ciencias Ambientales y Desarrollo Sostenible, Director de Investigación y Proyección Social de la Universidad Continental. 


\section{ABSTRACT}

Objectives: Determine the primary economic activities predominant in Huancaya, Alis and Vitis districts in Yauyos province, Lima region, located in the direct exploitation area of Nor Yauyos Cochas landscape reserve. Methods: It is an investigation of basic type, descriptive scope, cross-sectional design, in the process of data collection it was applied a structure questionnarie to a non-probability sampling of 79 people major of 18 years ald between men and women of Huancaya, Alis and Vitis districts, who participated in an academic event about "ecological business for development" organized by the "Econegocios" Social Projection Group of the Continental University, this same instrument was applied on persons circumstantially located in the public path, of the mentioned districts until to complete the sample size determination of 79 . Results: The $42 \%$ manifest that they are mainly dedicated on agriculture, $37 \%$ on cattle raising, $5 \%$ on aquaculture, $5 \%$ on crafts and $11 \%$ on other activities between can be identified the trade, touristic services, mining, etc. People whose mainly activity is agriculture manifested that $44 \%$ of its production is maize, $28 \%$ potatoes, $23 \%$ wheat and $5 \%$ other products. People dedicated to cattle raising manifested that $41 \%$ raise camelids, $28 \%$ bovine, $14 \%$ ovine, and $17 \%$ others. Conclusions: The population of Huancaya, Alis and Vitis districts are dedicated on the agriculture as one of the economic activities with major

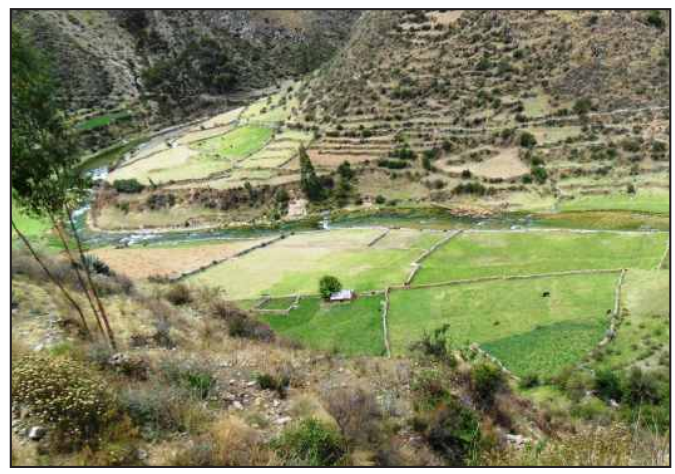

Figura 1. Reserva Paisajística Nor Yauyos Cochas, paisaje típico con áreas agrícolas, camino Vitis - Huancaya. predominance, then the cattle raising, these is an important indicator that characterizes these districts like zones with an economy based on primary activities, it means, that is an extractive economy of natural resources and use mainly for self-consumption, and for commercialization.

Key words: Economic activities, predominant, landscape reserve, Nor Yauyos Cochas, Huancaya, Alis, Vitis.

\section{INTRODUCCIÓN}

Del tema de investigación no existen estudios específicos pero si se encuentran trabajos relacionados con el tema.

Según el Plan Maestro de la Reserva Paisajística Nor Yauyos Cochas (RPNYC) menciona respecto a sus objetivos y actividades económicas lo siguiente:

La Reserva Paisajística Nor Yauyos Cochas es la primera área natural protegida establecida con esta categoría. Tiene como objetivo proteger aquellos ecosistemas inmersos en un conjunto paisajístico de gran belleza y singularidad, coexistiendo en armoniosa relación con las actividades de las comunidades campesinas, las cuales han desarrollado formas de organización social para la producción y uso eficiente de sus recursos naturales. La RPNYC se encuentra ubicada en la región andina central del Perú, entre las regiones de Lima y Junín, en las cuencas del Alto Cañete (Nor Yauyos) y del Cochas, Pachacayo.

Actividad agrícola: La actividad agrícola dentro de los distritos de Huancaya, Alis y Vitis es de desarrollo limitado y de alto riesgo por las variaciones climáticas propias de cada piso ecológico, ya que se realiza principalmente entre los 3000 a 3600 msnm, con gran presencia de laderas moderadamente empinadas, andenes y en algunos casos terrenos planos. Es muy poca el área cultivada que cuenta con riego, la mayor parte de los cultivos se siembran en 
secano.

Los principales cultivos, en orden de importancia, identificados en los distritos de Huancaya, Alis y Vitis según lo investigado son: maíz, papa, haba, oca, olluco, trigo, cebada, quinua, mashua, plantas medicinales y aromáticas; también los de destino pecuario como alfalfa, pastos cultivados, cebada y otros.

Actividad pecuaria: La actividad pecuaria dentro de los distritos de Huancaya, Alis y Vitis, es importante porque está articulada al mercado local y genera los mayores ingresos para las poblaciones de la zona de estudio. Su importancia radica en que los productos y subproductos como carne, lana, leche, etc. no sólo se destinan para el consumo local, sino también se ofertan a los mercados regionales.

En los distritos de Huancaya, Alis y Vitis, por sus características climáticas es propicia para la producción intensiva del ganado, en especial vacuno, ovino y en las partes bajas los caprinos. En la zona alta entre los 3900 y 4500 msnm, la actividad económica se centra en la actividad pecuaria, principalmente ganado, camélido y ovino.
En los distritos de Huancaya, Alis y Vitis, la crianza de ovinos es la principal, la cual se destina básicamente a la producción de carne. Este ganado tiene unos niveles de rendimiento bajos y no muy alta calidad de lana. La lana se oferta internamente a través de ferias y la carne se destina al abastecimiento de mercados locales. En general, la alimentación del ganado se basa en pastos nativos, cultivados, alfalfa, alimentos concentrados y el maíz chala. (1)

La actividad ganadera en la RPNYC se fundamenta en la explotación de sus pastos naturales, los cuales soportan a una ganadería conformada principalmente por ovinos, camelidos y vacunos.

La explotacion de ovinos es la actividad más importante en la RPNYC. El tipo de ganado esta constituido mayormente por "criollos" o "huacchas", que se caracterizan por no tener una conformación fenotipica ni genotipica uniforme; sin embargo, son de alta rusticidad. La crianza de Alpacas es una actividad importante y se lleva a cabo mayormente sin ninguna tecnificación en su explotación, a excepcion de la empresa SAIS "Tupac Amaru". La explotación de las Llamas está concentrada encima de los 3

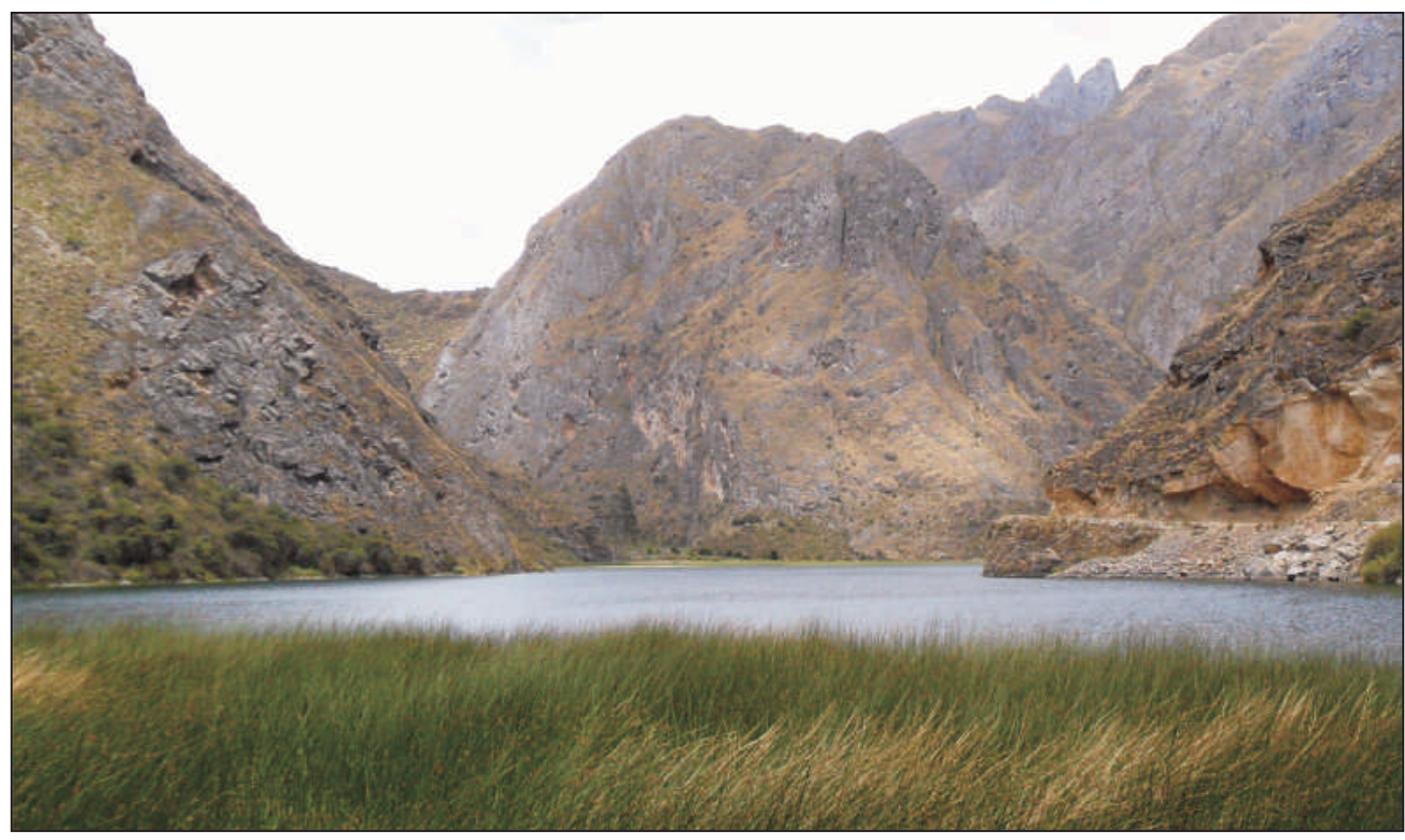

Figura 2. Reserva Paisajística Nor Yauyos Cochas, humedales altoandinos, camino Alis - Huancaya. 
800 msnm. Son utilizadas como animales de carga y productores de carne (fresca y seca). La explotación de los Vacunos se localiza debajo de los 4000 msnm. Los ingresos principales que genera esta actividad provienen de la vaca, que es comercializada en pie y sacrificada en camales de Cañete, Lurin y Lima. Igualmente, genera ingresos por la venta de leche y quesos. (2)

Actividad piscícola: La actividad piscícola que se puede considerar como de extracción pecuaria, es la pesquera. Esta se realiza, ya sea con la crianza en piscigranjas de truchas instaladas en algunas comunidades o a través de extracción directa del río. Dentro del ámbito del Área Natural Protegida (ANP).

Actividad turística: Esta actividad dentro de los distritos de Huancaya, Alis y Vitis, presenta un conjunto paisajístico de mucha relevancia y singularidad, que le otorga un enorme potencial recreativo y turístico a la zona. Se han registrado actividad turística por grupos de visitantes nacionales y extranjeros que se han desplazado a la zona en los últimos años, tanto desde Lima como desde la ciudad de Huancayo.

Las agencias turísticas que operan en la zona no promocionan el área como un destino turístico en su conjunto, solo se oferta algunos recursos turísticos, ocasionando que la actividad turística se concentre en zonas; estando sesgado este flujo principalmente al nevado Pariacaca (Tanta), bosque de Vilca, lagunas y saltos de agua formados por el río Cañete en Huancaya y Vitis; zonas en las que se ofertan las actividades turísticas de trekking, observación de paisajes, flora y fauna y pesca deportiva.

Las vías y las rutas de acceso permiten el desplazamiento hacia el área protegida y luego al interior de la misma, sin embargo esta red se encuentra en mal estado, el servicio de transporte es deficiente, especialmente para movilizarse dentro de ella. Asimismo, en la mayoría de los casos no se cuenta con senderos definidos para visitar los recursos turísticos ni señalización para llegar a ellos; dificultando un adecuado desarrollo turístico del área. El servicio de alojamiento dentro del área tiene una capacidad instalada de 18 hospedajes que funcionan permanentemente, con una capacidad de 270 camas, servicios higiénicos comunitarios y agua caliente en la mayoría de los casos. Cabe resaltar, que

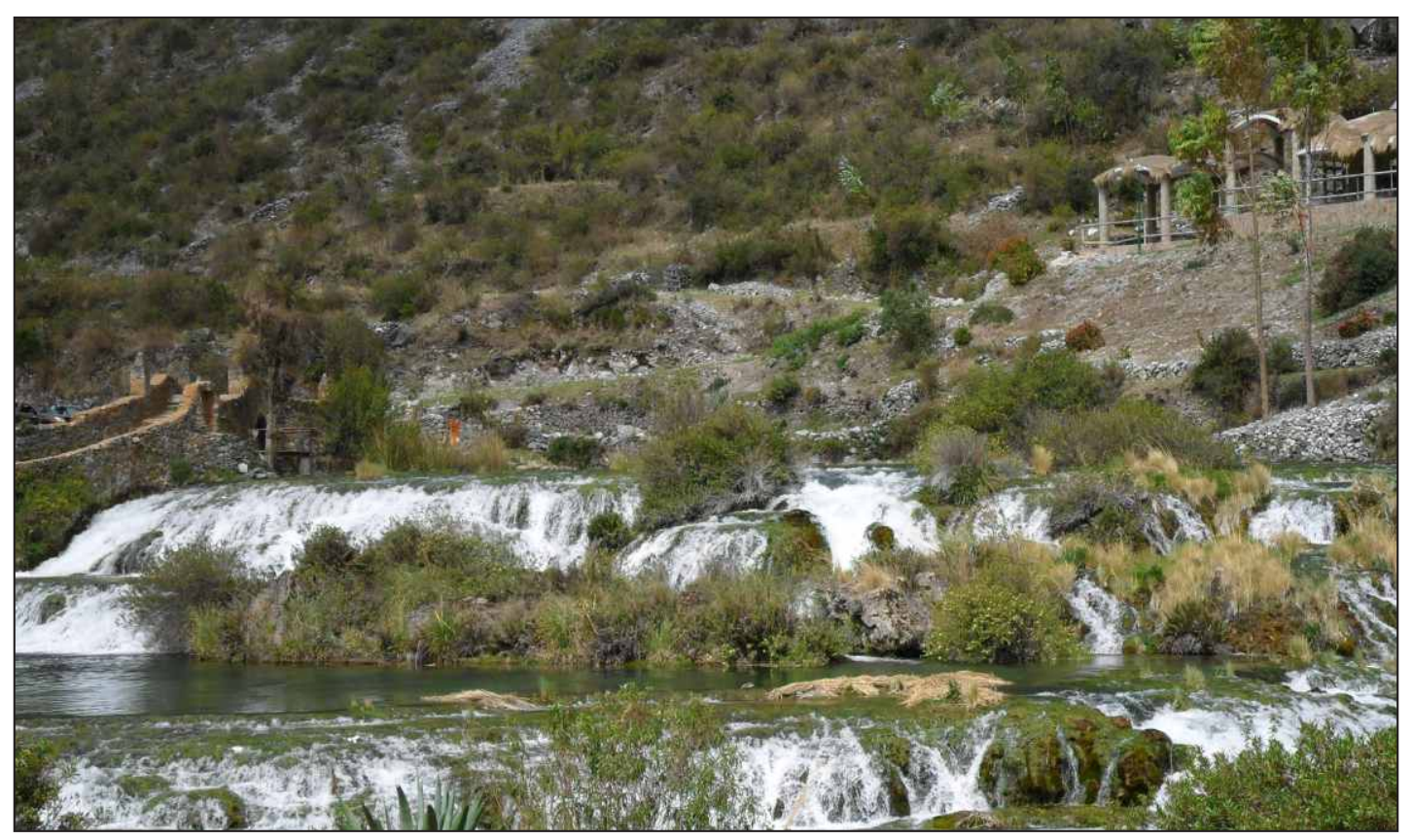

Figura 3. Reserva Paisajística Nor Yauyos Cochas, cascadas en Huancaya. 
estos servicios no cumplen con los estándares de calidad que demandan los visitantes. A estos se suman una cantidad no determinada de hospedajes ocasionales, a los que es necesario registrar y evaluar. (1)

El adecuado uso de los recursos naturales renovables nos ofrece muchas oportunidades en los eco-negocios. En esta área nos encontramos con tres sistemas diferentes. En un extremo se observa una agricultura moderna, eficiente, competitiva e integrada al mercado internacional, que no necesita prácticamente de apoyo por parte del Estado. En esta área se encuentran

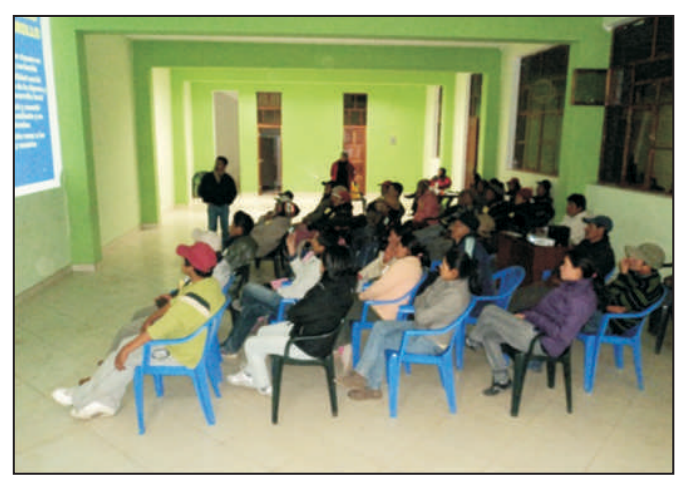

Figura 4. Reserva Paisajística Nor Yauyos Cochas, reunión de capacitación y recolección de datos.

productos como los espárragos, hortalizas y algunos frutales. En el otro extremo se encuentra el escenario de la agricultura de subsistencia, que hace uso fundamentalmente de la chaquitaclla, se ubica en las poblaciones alto andinas y no está articulada al mercado. En el centro de estos dos extremos se ubica la pequeña y mediana agricultura, sectores que con una economía apenas rentable subsisten a pesar de la escasez de agua, la falta de crédito y de asistencia técnica y con dificultades de acceso al mercado por falta de canales de distribución. (3)

Según el INRENA, entre todas las actividades económicas vinculadas con el turismo, tales como establecimientos comerciales, hospedaje y de alimentación se desarrollan en mayor cantidad en el distrito de Huancaya (4).
De acuerdo a la Municipalidad Distrital de Huancaya, uno de los distritos objeto de estudio, la estructura productiva Huancayana está dominada por la actividad agropecuaria, destacándose la ganadería. En los últimos años se ha notado un interés por el desarrollo turístico y sus actividades conexas como la artesanía, porque el distrito esta insertada dentro de la Reserva Paisajística Nor Yauyos Cochas. La PEA agropecuaria, representa un $58.5 \%$ de la PEA, destacando que la PEA femenina es un $45 \%$; cuya producción está destinada para el consumo (productos de pan llevar), y el mercado local, regional y nacional. (5) En el ámbito del turismo, Huancaya se define como una comunidad campesina dedicada a la ganadería, agricultura y el turismo como principales actividades económicas. (6)

La problemática identificada, para efectos de este estudio fue la necesidad de caracterizar a los distritos de Huancaya, Alis y Vitis respecto a las actividades económicas primarias predominantes, para que a partir de ello se pueda intervenir con programas de responsabilidad social, se tiene información general sobre diversas actividades económicas a nivel de la RPNYC, sin embargo no existen en detalle por distritos.

El objetivo fue determinar las actividades económicas primarias predominantes en los distritos de Huancaya, Vitis y Alis, ubicadas en la zona de aprovechamiento directo de la Reserva Paisajística Nor Yauyos Cochas, para efectos de implementar a futuro un programa de capacitación prioritariamente vinculada a las actividades económicas que predomina en las zonas de estudio; este programa sería un proyecto social de la Universidad Continental desarrollado como parte de los esfuerzos de la universidad para preservar los recursos naturales y conservar el medio ambiente generando un desarrollo sostenible en comunidades pobres económicamente tales como las de la RPNYC.

Asimismo los resultados de esta investigación permitirá focalizar adecuadamente intervenciones sociales del gobierno o de organizaciones del sector público o privado. 


\section{MATERIAL Y MÉTODOS}

Investigación básica, de alcance descriptivo y diseño no experimental transversal, en la recopilación de datos se utilizó un instrumento estructurado de tipo cuestionario.

La población a partir de la cual se recopilado la información ha estado compuesta por pobladores mayores de 17 años, varones y mujeres de los distritos de Huancaya 484 personas, Alis 380 y Vitis 494 personas, haciendo un total de 1358 personas, según datos obtenidos del Censo 2007 (Censo X Población y $\mathrm{V}$ de Vivienda) del Instituto Nacional de Estadísticas e Informática (INEI).

La muestra es no probabilística, constituida por 79 personas mayores de edad, seleccionados circunstancialmente a quienes se aplico un cuestionario en el proceso de asistencia a un evento académico y otros que fueron entrevistados en la vía pública de los tres mencionados distritos.

Los materiales utilizados son: equipo fotográfico, cuaderno de campo y fichas de registro de información.

\section{RESULTADOS}

Entre los hallazgos de este estudio se puede mencionar que más del $84 \%$ se desempeña en alguna de las actividades económicas primarias de agricultura, ganadería, acuicultura o mineria en la zona de aprovechamiento directo de la Reserva Paisajística Nor Yauyos Cochas.

Es evidente que por los recursos naturales existentes y las condiciones sociales en la cuales viven las personas de estos distritos, la mayoría de la población económicamente activa realizan alguna actividad relacionada a la Agricultura y Ganadería, figura 5.

Respecto a los cultivos de mayor

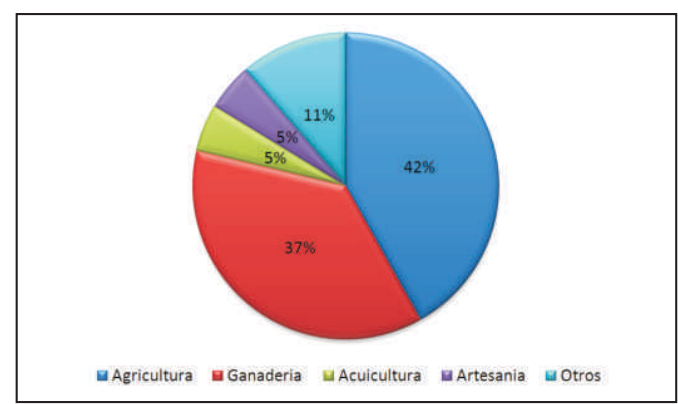

Figura 5. Actividades Económicas predominantes en la Reserva Paisajística Nor Yauyos Cochas.

predominancia, los encuestados manifestaron que la mayoría, más del $72 \%$ produce maíz y papa, muy pocos producen papa orgánica, luego se tiene productos como el trigo, cebada, entre otros. También un $51 \%$ de los pobladores que se dedican a la agricultura utilizan su producción para el autoconsumo, 38\% para la venta local y $8 \%$ para la venta regional. Asimismo, el $49 \%$ de los pobladores que se dedican a la agricultura usan los pastos naturales para forraje (pecuario), 33\% con fines comerciales y ningún uso un 18\%, figura 6.

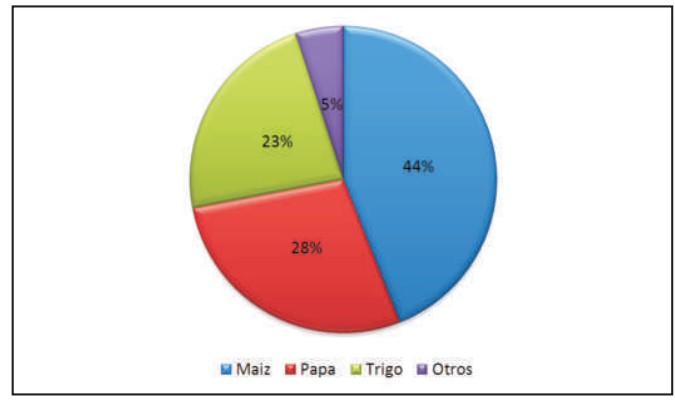

Figura 6: Cultivos predominantes en las personas dedicadas a la Agricultura en la RPNYC, distritos de Huancaya, Alis y Vitis.

Las personas dedicadas a la ganadería manifiestan que, un $41 \%$ cria camélidos, éstos alimentados casi en su totalidad por pastos naturales, $28 \%$ cría vacunos entre raza pura y criolla, $14 \%$ ovinos, y un $17 \%$ otros. En el grupo de otros se considera también a aquellas personas que manifestaron que crian truchas en piscigranjas (con agua de ríos y lagunas), que es el $9 \%$., pese al apoyo que poseen de las municipalidades en ésta actividad. Se cuenta con piscigranjas (pozos) y jaulas 


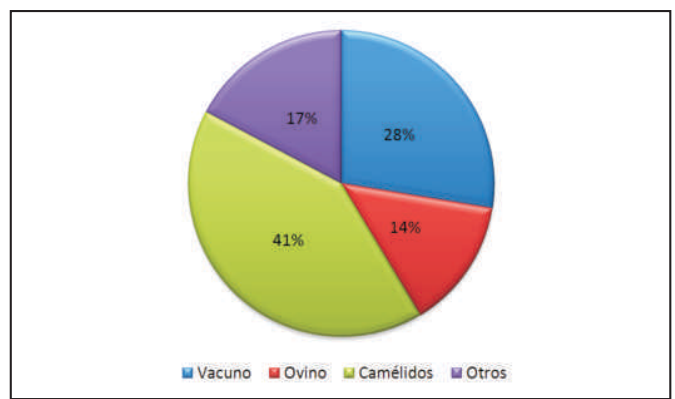

Figura 7. Crianza de ganado predominante en las personas dedicadas a la Ganadería en la RPNYC, distritos de Huancaya, Alis y Vitis.

instaladas en lagunas.

Las personas que se dedican a la artesanía generalmente producen productos tejidos y cerámica, solo para la venta a los turistas que visitan estos distritos.

Finalmente, el género y edad de los entrevistados fue de $61 \%$ mujeres y el $39 \%$ varones, $72 \%$ entre 18 y 30 años y el resto de 30 años a más.

\section{DISCUSIÓN}

De acuerdo al INRENA (hoy parte del Ministerio del Ambiente) los principales cultivos, en orden de importancia, identificados en los distritos de Huancaya, Alis y Vitis son: maíz, papa, haba, oca, olluco, trigo, cebada, quinua, mashua, entre otros; también los de destino pecuario como alfalfa, pastos cultivados, cebada y otros. (1) De acuerdo a nuestro estudio los productos de mayor cultivo son el maíz y la papa, en tal sentido existen coincidencias y se puede reafirmar los hallazgos realizados en el estudio del INRENA.

Según el Ministerio del Ambiente, la crianza de Alpacas en la RPNYC es una actividad importante y se lleva a cabo mayormente sin ninguna tecnificación en su explotación, a excepcion de la empresa SAIS "Tupac Amaru". La explotación de las Llamas está concentrada encima de los 3800 msnm. Son utilizadas como animales de carga y productores de carne (fresca y seca). La explotación de los Vacunos se localiza debajo de los 4000 msnm. Los ingresos principales que genera esta actividad provienen de la vaca, que es comercializada en pie y sacrificada en camales de Cañete, Lurin y Lima. Igualmente, genera ingresos por la venta de leche y quesos. (2), confirmando de este modo nuestra investigación, respecto al tipo de ganado que se cria en los distritos de Huancaya, Alis y Vitis. Es importante mencionar que a nivel de toda la RPNYC la zona de mayor producción de ovinos es la zona de Cochas y Pachacayo.

Los estudios de Álvarez C. indican respecto a la agricultura que, en un extremo se observa una agricultura moderna, eficiente, competitiva e integrada al mercado internacional, que no necesita prácticamente de apoyo por parte del Estado. En esta área se encuentran productos como los espárragos, hortalizas y algunos frutales. En el otro extremo se encuentra el escenario de la agricultura de subsistencia, que hace uso fundamentalmente de la chaquitaclla, se ubica en las poblaciones alto andinas y no está articulada al mercado. En el centro de estos dos extremos se ubica la pequeña y mediana agricultura, sectores que con una economía apenas rentable subsisten a pesar de la escasez de agua, la falta de crédito y de asistencia técnica y con dificultades de acceso al mercado por falta de canales de distribución. (3) En este apartado se pone de manifiesto que estas zonas donde existe mucha pobreza un medio de subsistencia es la agricultura y tiene las características a las cuales hace mención Álvarez C., es decir utilizan tecnología del inkanato con bajos niveles de productividad y generalmente para autoconsumo.

De acuerdo a la caracterización de las actividades económicas predominante en el distrito de Huancaya, la Municipalidad de Huancaya menciona a través de su sitio web que la agricultura y ganadería son los ejes de la economía local, coincidiendo con nuestro estudio.

En conclusión, los distritos de Huancaya, Alis y Vitis que se encuentran en la zona de 
aprovechamiento directo de la RPNYC se caracterizan por tener una economía basada en actividades económicas primarias, siendo las actividades en la cual un alto porcentaje de la población esta involucrada la agricultura y ganadería.

\section{Agradecimientos}

A los estudiantes del Grupo de Proyección Social "Eco-negocios" de la Universidad Continental, integrado por los estudiantes Erold Quispe Fuentes, Dyada Jeremias Yauri, Elisa Alfaro Ramos, Alberto Yallico Amaya, Roxana Arrece Toro, Angel Guere Canqui, Karina Maximiliano Melgar, Sofia Mieses Castillo, Daniela Palomino Venturo, Jherson Poma Bravo, Diego Ninamango Jaime, Guisela Rodriguez Valladares, Edith Surichaqui Rodríguez, Diana Sanabria Granados, María del Carmen Carbajal Vergara, Lucero Limachi Calderón, Diana Torres Alanya, Pamela Huamán Rosas, Lourdes Alva Meza y Noelia Salazar Salome; quienes contribuyeron en la recolección de datos con dos viajes quincenales realizados desde la ciudad de Huancayo. 


\section{REFERENCIAS BIBLIOGRAFICAS}

1. Instituto Nacional de Recursos Naturales - INRENA. Reserva Paisajística Nor Yauyos Cochas, Plan Maestro 2006 - 2011 . Lima: INRENA; 2006.

2. Dirección General de Evaluación, Valoración y Financiamiento del Patrimonio Natural del Ministerio del Ambiente. Inventario y evaluación del patrimonio natural en la Reserva Paisajística Nor Yauyos Cochas. $1^{\circ}$ Edición. Lima: Ministerio del Ambiente; 2011.

3. Álvarez C. Riqueza Natural de la Nación: La Gran Paradoja. Lima: Ediciones Torre Azul; 2004.

4. Instituto Nacional de Recursos Naturales - INRENA. Plan de uso turístico y recreativo de la RPNYC 2008 - 2012. $1^{\circ}$ Edición. Lima: INRENA; 2007.

5. Municipalidad Distrital de Huancaya [Internet]. Huancaya: Municipalidad Distrital de Huancaya; 2012 [citado el 01/02/12]. Disponible en: http://www.huancaya.lim. md.gob.pe/potencial_economico.html

6. Pakary Travel, Grupo Turístico [Internet]. Huancaya: Pakary Travel; 2012 [citado el $01 / 02 / 12]$. Disponible en: http://www.pakarytravel.com/index.php ?option $=$ com_content\&view $=$ article\&id $=71 \&$ ltemid $=56$

Correo electrónico:

wbulege@continental.edu.pe 